\title{
La investigación histórica en los proyectos de gestión del patrimonio construido. Propuesta metodológica
}

\author{
Jorge Rolando García Perdigón \\ Historiador especialista en Museología \\ Master en Estudios interdisciplinarios sobre \\ América Latina, el Caribe y Cuba \\ jorge@bellasartes.co.cu
}

\section{Resumen}

El presente estudio, realizado en el bienio 2007-2008 como tesis de maestría, define una propuesta metodológica para la realización de una investigación histórica en los proyectos de gestión y manejo del patrimonio edificado. Constituyendo un importante aporte, dado que las fuentes estudiadas hasta el momento solo se quedaban en brindar una guía metodológica solamente a la hora de intervenir en una edificación.

Se ejemplifica su necesidad tomando como caso específico el antiguo Convento de Santa Clara de Asís, a la luz de su restauración para sede del Centro Nacional de Conservación, Restauración y Museología (Cencrem), y como edificación patrimonial donde no se aplicó correctamente el examen histórico en su proyecto de gestión. Además, la elaboración de esta investigación sobre los procedimientos metodológicos, resolvería problemas comunes a otras realidades presentes en Cuba y Latinoamérica.

La propuesta parte de la definición del objeto de estudio, según la clasificación del patrimonio cultural dado por la legislación vigente, analiza la situación del estado informativo que se tiene sobre el mismo, para después realizar la investigación propiamente dicha siguiendo un orden cronológico. Finalizando con la elaboración del informe de los resultados obtenidos, para completar la base de datos históricos sobre el objeto tratado. Queda así definido, del modo más acabado posible, el acercamiento investigativo propuesto.

Palabras claves: estudios históricos, gestión del patrimonio, patrimonio edificado, propuesta metodológica. 


\begin{abstract}
This study, conducted in 2007-2008 as master's thesis, defines a methodology for conducting research on the maintenance of historical buildings. It makes an important contribution since the methodology studied so far only offer guidelines for intervention.

It shows the need to take as a specific case the former Convent of Santa Clara de Asis, restored to host the National Center for Conservation, Restoration and Museology (Centro Nacional para la Conservación, Restoración y Museología, Cencrem, given its Spanish acronym), as a heritage building project where a proper historical review was not conducted correctly. In addition, the advancement of research about such procedures would resolve similar problems also present in Cuba and Latin America.

Part of the objective of the study, according to the classification given by cultural heritage laws, is to analyze the current state of information about the subject and then perform the necessary research in chronological order. It will end with a report about the results obtained in order to complete the historical database on the subject. Thus defined and in the most exhaustive manner possible, the investigation hopes to reconcile the various issues.
\end{abstract}

Keywords: historical studies, heritage management, building heritage, methodological proposal.

\title{
Introducción
}

El conocimiento de la historia y sus bondades constituye el punto de partida para toda operación que se debe aplicar al patrimonio histórico y cultural. Proteger, conservar y poner en valor ese patrimonio es hoy obligación y compromiso de futuro no solo para las instancias públicas, sino para el pueblo todo en su conjunto, ya que en tal acervo se encuentra su propia esencia como colectivo.

La revalorización del patrimonio cultural junto con la regeneración y el desarrollo sostenible de los centros históricos constituyen prioridades presentes en las agendas programáticas de las autoridades locales de las ciudades de América Latina y el Caribe. Aunque es justo reconocer que tal actitud es un punto cada vez más frecuente en los programas y acciones de gestión estatal y gubernamental en otras regiones del mundo.

A pesar de la diversidad de contextos económicos, sociopolíticos y urbanos, los barrios antiguos y edificaciones patrimoniales de las ciudades de la región latinoamericana presentan problemáticas comunes, que ponen en evidencia la necesidad de analizar las políticas de protección y gestión de su patrimonio para encontrar soluciones. 
El Consejo Nacional de Patrimonio Cultural (CNPC), del Ministerio de Cultura de la República de Cuba, es la instancia encargada de precisar y declarar los bienes que deben formar parte del patrimonio cultural de la nación, los cuales están sujetos a los preceptos de la Ley n. ${ }^{\circ}$, Ley de Protección al Patrimonio Cultural, aprobada por la Asamblea Nacional del Poder Popular el 4 de agosto de 1977 y su Reglamento, el decreto n. ${ }^{\circ} 118$ del Consejo de Ministros del 3 de noviembre de 1983.

Para el sistema de protección y conservación del patrimonio construido no existe una metodología en el enfrentamiento de los análisis históricos en los proyectos de gestión. Hasta el presente estos estudios se ejecutan siguiendo criterios generales de la investigación histórica que no se adaptan, en su totalidad, al contexto de intervención en los entornos construidos. De ahí nuestro propósito y aporte, al realizar un examen integral que sitúe a los estudios históricos como pieza fundamental en el proceso de gestión cultural del patrimonio en cuestión.

Conceptualmente la investigación histórica se presenta como la búsqueda crítica de la verdad que sustenta los acontecimientos del pasado, y al gestionar el patrimonio nos estamos refiriendo al conjunto de acciones destinadas a hacer efectivo su conocimiento, conservación y difusión, lo que incluye ordenar y facilitar las intervenciones que en él se realicen. Los métodos que se han de utilizar serán el analítico-sintético y el deductivo-inductivo, al encontrar lo nuevo e interpretarlo, sacando sus consecuencias mediante el razonamiento.

Dada la importancia y necesaria actualización de los razonamientos históricos en la gestión del patrimonio construido, esta temática adquiere un interés relevante. Se trata de analizar la necesidad de estas reflexiones en el contexto cubano actual, para el logro de resultados mucho más cercanos a la obtención de los objetivos en la intervención constructiva sobre los inmuebles o conjuntos inmobiliarios. Estas memorias y sus resultados, conllevarán al cumplimiento de la premisa más importante de toda intervención, el logro de la autenticidad respetada o rescatada en el bien intervenido.

Es objeto de estudio de nuestro examen la definición de una metodología que tenga en cuenta la importancia de la investigación histórica en el proceso de gestión y manejo de un bien inmueble, ejemplificando su necesidad con el caso específico del antiguo Convento de Santa Clara de Asís, a la luz de su restauración para sede del ya desaparecido Centro Nacional de Conservación, Restauración y Museología (Cencrem).

Basamos esta selección como muestra teniendo en cuenta que fue el primer caso en Cuba de un proyecto integral de intervención para la recuperación de un monumento, en aras de un programa de gestión sociocultural y haber constituido un ejemplo de estudio histórico incompleto. Además, es interés del Programa Ramal de Investigaciones del Ministerio de Cultura (Mincult) la elaboración de 
esta investigación sobre los procedimientos metodológicos, para resolver estos problemas, comunes a otras realidades presentes en el país.

A partir de la definición del objeto de estudio, es válido cuestionarnos la necesidad de los estudios históricos en el proceso de gestión del patrimonio construido y la posibilidad de definir una metodología con base en esas memorias, tomando el ejemplo del Convento de Santa Clara de Asís como edificación patrimonial donde no se aplicó correctamente el examen histórico en su proyecto de gestión.

Las formulaciones anteriores llevaron a plantearnos los objetivos siguientes:

1) Demostrar la necesidad de los estudios históricos en el proceso de gestión del patrimonio construido, mostrando su importancia cultural, social y económica.

2) Realizar una actualización histórica del Convento de Santa Clara de Asís, al constituir el principal referente de los deficientes estudios investigativos realizados en un proyecto de intervención.

3) Proponer una metodología general que tenga en cuenta las especificidades en los diferentes casos de la investigación histórica en la gestión y manejo cultural de un bien inmueble.

\section{Desarrollo}

Importancia de la investigación histórica para la toma de decisiones en la política de gestión que se diseñe para el edificio.

Entre lo estipulado por la Convención sobre la Protección del Patrimonio Mundial, Cultural y Natural de 1972, se destaca el desarrollo de los estudios y la investigación científica y técnica. Aspectos que proporcionarán al país hacer frente a los peligros que amenacen a su patrimonio, adoptando, para ello, las medidas jurídicas, científicas, técnicas, administrativas y financieras adecuadas que posibilitarían identificar, proteger, conservar, revalorizar y rehabilitar ese patrimonio. $^{1}$

La investigación histórica realizada a un edificio patrimonial, además de evaluarlo integralmente, facilita la toma de decisiones para una buena política de su gestión Al revelarnos su importancia, también nos muestra su estado general de conservación, sus características arquitectónicas, materiales utilizados, técnicas decorativas, usos y fines. Es la guía para la acción futura y elemento clave a respetar para lograr el éxito.

1 Pérez Cano, $\mathrm{M}^{\mathrm{a}}$ Teresa. "Turismo en las ciudades históricas: la ciudad sosteniple”. En Boletín del Instituto Andaluz del Patrimonio Histórico. n. ${ }^{\circ}$ 36, 2001.

www.juntadeandalucia.es/cultura/iaph/puplicaciones/dossiers/dossier11/dossier11art2.htm. 
El respeto por el patrimonio y la puesta en valor de las obras tal como se encuentran, aceptando las intervenciones incorporadas en el transcurso vital del edificio, han llevado a la necesidad de poseer un detallado conocimiento histórico de este antes de acometer tareas que lo afecten. En tiempos pasados, la restauración estaba sujeta a los caprichos del restaurador y el conocimiento histórico de la obra no se consideraba necesario.

Es en los últimos años del pasado siglo que se ha jerarquizado el respeto hacia el edificio y su entorno, a partir de lo reglamentado internacionalmente, y se ha acentuado la necesidad de realizar los trabajos de restauración sobre bases científicas. La documentación histórica se afianzó como un dato ineludible para la acción. Sin embargo, por razones coyunturales, las urgencias por comenzar las obras y las improvisaciones, entre otras, hace que, aún hoy, sea frecuente la realización de tareas de restauración prescindiendo del conocimiento histórico del objeto.

Estos ignorados estudios ofrecen un conjunto de informaciones que permiten dar validez a cualquier acción realizada en la gestión del patrimonio construido. Los monumentos deben ser analizados como documentos, reconocerle sus valores históricos y artísticos, antigüedad y todo lo que nuestros antepasados fueron capaces de crear y transmitir. Estas exposiciones requieren de un profesional altamente calificado, que realice un trabajo diferenciado, con una metodología específica y que participe en el gran debate teórico sobre los principios de restauración y revitalización del monumento.

Son diversas las formas de intervenir en la preservación y revalorización de los monumentos arquitectónicos, pero la validez de cualquier acción estará determinada por los resultados de las investigaciones que de ellos se realicen, por lo que dichas intervenciones aportarán en la definición de las medidas que se deben tomar para devolverle un estado adecuado para su uso.

Existen factores que condicionan las formas de intervenir en los monumentos, y su aplicación estará condicionada por las características especificas de la edificación en la que se va a intervenir y su entorno, según la documentación existente sobre este y según los objetivos que se persigan con la acción de conservación. ${ }^{2}$

Se deberá tener en cuenta el grado de permanencia de los componentes originales del monumento, entendiéndose por originales todos aquellos que correspondan a la etapa histórica que se tendrá que recuperar o que hayan aparecido en el transcurso de la evolución del edificio, mientras conservó el uso original, considerándose los componentes y demás bienes.

2 Cárdenas, Eliana. "La investigación histórica en los monumentos arquitectónicos, preservación y revalorización”. Arquitectura y urpanismo. ISPJAE, 1981, volumen II. Págs. 106-107. 
La realización de cualquier trabajo de conservación de un monumento no debe permitirse... sin antes haberse realizado una profunda labor de investigación histórica y arquitectónica, así como el resto de los estudios e investigaciones. ${ }^{3}$

También se reconoce el papel del historiador del arte en el proceso de restauración y revitalización, asociándolo no solo con tareas del inventario formal, sino también con el estudio histórico, estético y del estado de conservación del inmueble.

El conocimiento de la obra, en todas sus etapas históricas, facilitará la adopción de decisiones adecuadas sobre lo que se debe conservar y lo que se debe eliminar. Dominar la historia del monumento en cuestión “....no es un lujo de exquisitos sino una necesidad para quien encare responsablemente la preservación del patrimonio cultural...". ${ }^{4}$

Existen numerosos ejemplos, nacionales y extranjeros, donde puede evidenciarse la necesidad de vincular estos estudios con las formas de intervención. La experiencia indica que, estando en camino la restauración, el edificio nos presentará continuos interrogantes que la investigación podrá responder. Es por ello que, si bien esta debe ir por delante de la restauración, eso no significa que cumpla un ciclo cerrado, sino que, por el contrario, debe acompañar las fases del proceso de la obra.

Así, observamos restauraciones realizadas en los últimos años que, no obstante el esfuerzo resultan deficientes, y se han incorporado usos que, si bien como actividad no son desechables, sí lo es la forma en que se han ubicado. Precisamente ha sucedido en algunos de los elementos del sistema defensivo de La Habana colonial: La Chorrera y los castillos de los Tres Reyes del Morro y el de San Salvador de la Punta, en los que se han ejecutado acciones revitalizadoras que han dañado el monumento $y$, en algunos casos, pudieran resultar irreversibles. ${ }^{5}$

En los trabajos de impermeabilización ejecutados a los techos del Castillo del Morro se evidenciaron los canales de desagüe originales de la fortaleza, que correspondiendo a un sistema ingenioso bien pudiera haber sido elemento importante que se ha de mostrar como parte del proceso de restauración de la edificación. No se había hecho una intervención previa de este sistema desde el punto de vista documental, pues no se había encontrado información al respecto, ni se hizo desde el punto de vista arquitectónico en el momento de su hallazgo. Solo se tomaron algunas fotos y se volvió a cerrar, perdiéndose así una posibilidad importante de conocer el monumento y de dar una solución adecuada en los trabajos de conservación.

3 Ibíd. Pág. 114.

4 Viñueles, Graciela y Ramón Gutiérrez. "La documentación histórica en la restauración de monumentos". En Cuadernos de arquitectura y conservación del patrimonio artístico. Serie Ensayos n. 2, México, marzo de 1976. Pág. 19.

5 Cárdenas, Eliana. Op. cit. Pág. 114. 
Otro ejemplo de poca profundidad en el estudio de los valores reales del monumento, por parte de los restauradores, es el Castillo de la Punta. Aquí no se aplicaron las técnicas más adecuadas para la conservación, llegándose a una solución poco apropiada en las cubiertas, que fueron levantadas y no pudieron volverse a colocar, siendo sustituidas por una estructura de hormigón armado. De igual forma, otras soluciones en diferentes partes de la edificación no han sido las más adecuadas, evidenciando el poco cuidado necesario en la preservación de los valores de uno de los elementos fundamentales del sistema defensivo de La Habana, único en América por sus características y concentración.

En la obra de restauración de importantes monumentos en el Cusco, capital del Imperio inca e histórica del Perú, también se han cometido errores dignos de ser reflejados en este trabajo. En San Bernardo, la coordinación incorrecta de los documentos existentes y lo que la obra presentaba, hizo caer a la oficina técnica en el error de hacer un diseño que contemplaba una escalera en una habitación que había servido de sacristía. Cuando fueron localizados los documentos del siglo XVIII se demostró que allí no había estado la escalera, debiendo cambiarse urgentemente el proyecto de restauración aprobado.

Hace algunas décadas se escribió que el templo del Convento-hospital de la Almudena databa de 1886, fecha que solo indicaba la erección de la primera capilla. Afirmación en la que se continuó creyendo hasta 1975, en que una investigación de la documentación existente en varios archivos, si bien confirmó la primera fecha, también mostró las vicisitudes acaecidas al edificio, la fecha de comienzo y terminación de la actual iglesia y el sitio original de su emplazamiento perpendicular al anterior.

Cuando se trabajaba en la obra del Seminario de San Antonio Abad se localizaron, enterradas, las piezas de una antigua fuente con una inscripción en parte borrada. Personas que se creían versadas en la Biblia completaron la leyenda a su modo y cerraron la búsqueda informativa, sin haber indagado que en el Museo del Arzobispado del Cusco se encontraba un cuadro que mostraba la fuente en todos sus detalles y con la inscripción completa. ${ }^{6}$

Esto nos refleja que si el arquitecto, al hallarse ante tal enigma, hubiera consultado al investigador para que buscara el documento adecuado se hubiera evitado una interpretación incorrecta, así como la demora y errores innecesarios.

Existen, sin embargo, ejemplos positivos donde la realización de una correcta investigación ha posibilitado no cometer errores o enmendarlos, rescatando el patrimonio para el disfrute social. En una de las casas más antiguas del país, el Museo de Ambiente Colonial de Santiago de Cuba, conociéndose el valor de los alfarjes...

6 Ver Viñueles, Graciela y Gutiérrez, Ramón. Op. cit. pp. 8-10. 
Se procedió a un trabajo de consolidación que permitía conservar las techumbres originales. Dado que los cabezales de las vigas estaban prácticamente podridos y y casino apoyaban en los muros, la consolidación se efectuó reforzando su asiento y engrosando ligeramente los muros, para que asi las vigas pudieran apoyar en los mismos y salvar la techumbre original, que de haberse levantado no hubiera podido recuperarse. ${ }^{7}$

En Barcelona, España, a través del Servicio del Patrimonio Arquitectónico Local (SPAL) de esa Diputación, se han efectuado desde los años 80 del pasado siglo importantes restauraciones del patrimonio arquitectónico local. Acciones desarrolladas mediante un estructurado proceso metodológico que se apoya en la investigación arqueológica, histórico-documental, histórico-artística e histórico-constructiva.

Fue así como los estudios arqueológicos detectaron el error inducido por las fuentes documentales en la datación de la iglesia de Sant Jaume Sesoliveres de Igualada. Dichas fuentes hablaban de la existencia de un templo en el siglo XI, la arqueología...

puso de manifiesto que el templo en cuestión no se habia levantado hasta finales del siglo XII o principios del XIII. Por otra parte, el análisis de su fábrica, tanto desde el punto de vista arqueológico como desde el históricoconstructivo, nos llevó a pensar que las cicatrices que mostraban los parámetros debian ser la huella de un movimiento sísmico que los desplomó y en parte los derribó, y también nos permitió hacer la lectura histórica de la reconstrucción del edificio a través de las soluciones constructivas que emplearon los albañiles del siglo XV para paliar los daños. ${ }^{8}$

También en esta iglesia, junto con la de Santa María de Castelledefels y la de Sant Quirse de Pedret, así como en el puente de esta última, en el medieval de Periques de Puig-reig y en el viejo de Roda de Ter,

...el análisis de la cartografía antigua, de grabados y dibujos paisajísticos, posibilitaron reconocer los antiguos caminos que daban sentido a la ubicación de cada uno de estos elementos en época medieval, las rutas que seguian, la actividad humana y económica que se derivaba de ellas: vías romanas, el camino real, el camino de Santiago, etc. ${ }^{9}$

Este conocimiento posibilitó rehacer las vías que accedían a los monumentos, ya desaparecidas o desdibujadas por la falta de uso.

7 Cárdenas, Eliana. Op. cit. (p. 112).

8 Lacuesta, Raquel. "Ó coñecemento histórico do monumento. Método e experiencias". En As actuacions no patrimonio construido: un diálogo interdisciplinar. Xunta de Galicia, 1997. (p. 420).

9 Ibíd. (p. 422). 
Todos estos ejemplos nos posibilitan afirmar la necesidad de ver a los estudios históricos como un todo, al facilitar reconstruir el devenir del patrimonio construido, su pasado, significado estético, sus lenguajes a través de generaciones. Tratan de mostrar la huella del hombre y el tiempo, de recomponer los pasajes y de darles a los monumentos, a ese patrimonio edificado, un sentido para la historia.

\section{Convento de Santa Clara de Asís}

Constituye el primer caso de un proyecto integral de intervención para la recuperación de un monumento en aras de un programa de gestión de esa magnitud que se realizó en Cuba. Situado en La Habana Vieja, Patrimonio de la Humanidad, es considerado uno de los edificios más antiguos del país, con un rico muestrario de formas arquitectónicas, siendo la primera estructura no militar de escala monumental que se construyó en la ciudad. Desde 1985 esta antigua edificación sirvió de sede al Cencrem, destacada institución destinada al desarrollo científico técnico y docente-metodológico de la conservación del patrimonio tangible, mueble e inmueble, y del patrimonio intangible.

A partir de él se comienzan a crear las bases de una posible estrategia cubana en las intervenciones para la restauración y conservación arquitectónica, incluyéndose los procesos de la investigación histórica y arqueológica. La complejidad de la intervención y la premura en la esfera de resultados concretos hicieron que los equipos de estudio y proyecto no utilizaran correctamente los estudios históricos, con criterios acordes a su época con los conocimientos que en ese momento se manejaban en el país y que no eran el de considerar al fenómeno de la restauración como proceso tecnológico que requiere de una amplia y sólida investigación científica por su propio carácter de transformación del objeto que se debía intervenir y su autenticidad.

La investigación histórica se dirigía para validar criterios de intervención y no para crear criterios en sí. Como ejemplos particulares pudiéramos citar estos: los cambios de pavimentos en galerías y locales, la uniformidad impuesta a las soluciones de ventanas y puertas, la aplicación de pintura azul habana en los techos y carpintería en general, la transformación de los espacios en el llamado Callejón de los Suspiros y otros ejemplos más.

El mantener ese proceso de diseño, desde esa fecha hasta hoy, hace que el Convento sea un exponente de problemas al cual el actual proceso de intervención que se realiza en la institución tenga que dar solución.

La realización de un análisis crítico de la investigación histórica dentro del proyecto de intervención del edificio contempla, primero que todo, delimitar los aspectos que quedaron deficientes en los anteriores estudios realizados, investigaciones caracterizadas de escuetas, plagadas de inexactitudes, datos sin 
referencia y fundamentación científica, lo que llevó a la toma de decisiones incorrectas.

Documentar la historia de estos edificios y de las órdenes religiosas que vivieron allí es una tarea difícil por las lagunas que caracterizan al registro histórico de nuestra ciudad, fundamentalmente de los siglos XVI al XVIII. Razón por la cual, la lectura del registro arqueológico es sumamente importante para establecer la utilización del espacio donde estas obras se alzan, en momentos previos a su edificación y en relación con sus etapas de construcción, ampliaciones y reparaciones. Importante también resulta la reconstrucción de elementos de la vida cotidiana que definen estilos de vida en el interior de estos magníficos inmuebles.

La inmediatez y premura en el comienzo de los trabajos de restauración del convento, en 1982, conllevó a la formación de un expediente que carecía de la documentación histórica suficiente y de un pobre apoyo arqueológico. Investigaciones desarrolladas posteriormente posibilitaron enmendar numerosos errores históricos que hasta ese momento se tenían como valederos.

Pienso que en estos errores influyó el guiarse por indagaciones anteriores realizadas sobre el edificio, carentes de fundamento histórico. Fe de esto son los libros Mis doce primeros años (1831) e Historia de sor Inés (1832), escritos y publicados en París por la condesa de Merlín, al recrear fantasiosas historias sobre el edificio conventual. Además de la obra Historia del Convento de Santa Clara de Asís, datos históricos y leyendas (1922), escrita por los periodistas Waldo Lamas y Osvaldo Valdés, donde, al valorar la presencia de la arquitectura cubana en la etapa colonial, también reflejaron errores históricos sobre la antigua edificación.

Fue el reconocido investigador Pedro Antonio Herrera, trabajador del Cencrem hasta su jubilación, quien dedicara largos años a la búsqueda de la documentación necesaria que posibilitara desentrañar errores y fantasías, permitiendo comprender en toda su dimensión la vida de este monumento. Fruto de su minucioso trabajo es el libro El Convento de Santa Clara de La Habana Vieja, publicado por la institución en el año 2006.

Para los equipos precedentes de investigación (1980-2006), la administración, control de ciclos y procesos cuyo alcance termina con la ejecución de la obra, la gestión no se ve como una continuidad sistémica sobre el objeto intervenido, lo que incluye su reelaboración conceptual, o sea, un cambio constante en el objeto que se mantiene más allá de la terminación de las acciones constructivas.

La investigación recorre el método de consulta y comparación de fuentes, y se deja al criterio del arquitecto jefe la toma de decisiones, dando como válidas las conclusiones del investigador. Se mantienen criterios vagamente fundamentados, alegándose su factibilidad a un proyecto muy del presente y no 
profundizando en los cambios cronológicos sobre el significado y las propias soluciones tecnológicas y funcionales de un inmueble que ha recorrido más de 350 años de existencia y en los últimos 80 años ha visto cambiar drásticamente su objeto de uso.

La valía de utilizar el antiguo Convento de Santa Clara como ejemplo principal de aplicación de estos procesos (antes y ahora) radica en la comparación que se genera al analizar los resultados en un caso paradigmático que sirvió de modelo para todos los procesos que le siguieron, incluyendo su utilización como método y ejemplo para los programas de capacitación y superación técnica y profesional que se han impartido en el Cencrem desde 1986 hasta la actualidad y que han impuesto una visión específica de abordar el problema de la investigación, que requiere de una lógica actualización.

La investigación histórica, usada tradicionalmente para la validación del bien como patrimonio y categorizarlo según su importancia (mundial, nacional, local), ahora es también punto de partida obligado para analizar comportamientos, impactos, en aspectos como la tecnología, los ambientes circundantes, la población; con cronologías sugerentes para cada una de estas cuestiones de análisis.

El ejemplo expuesto, junto con otros aquí referidos, afirman la necesidad de ver a los estudios históricos en su sentido holístico, al posibilitar reconstruir no solo el devenir del patrimonio construido, su pasado, significado estético y sus lenguajes a través de generaciones, sino también sus contextos en el horizonte del arte, la ciencia y la tecnología, lo cual posibilita realmente una reconstrucción y un análisis de todos los elementos que conforman el proyecto de intervención. También justifican el fin del desarrollo investigativo signado por los procesos de inventario y registro esquemático, característica que aún rige la labor de los equipos de intervención y que mutilan la potencialidad de la ciencia histórica en su aplicación en la gestión y manejo del patrimonio construido.

\section{Propuesta metodológica}

Una vez analizada la experiencia anterior, estamos en condiciones de dejar definida una propuesta teórico-metodológica para suplir las deficiencias detectadas y guiar a los futuros equipos de proyectos. Es un método que define como válidos cuatro pasos para enfrentar una experiencia investigativa sobre temas análogos o similares.

1) Definición del objeto de estudio, según la clasificación del patrimonio cultural dado por la legislación vigente, así:

- Objeto edilicio. Edificio aislado, conjunto edificado, monumento o ruina arqueológica.

- Objeto urbano. Sitio o centro histórico urbano. 
- Objeto paisaje cultural. Territorio natural o rural.

Al definir y clasificar el objeto de estudio, el investigador se ubica en el ente patrimonial que se debe analizar, conociendo su ubicación y características principales.

2) Conocimiento del estado de la información sobre el objeto de estudio, en coherencia con el objetivo de la intervención.

- Existencia de investigaciones precedentes.

- Existencia de documentación escrita y gráfica.

- Bibliografía editada (plana).

- Bibliografía inédita.

- Bibliografía en soporte digital y on line.

- Existencia de tradiciones y conocimientos orales e intangibles.

- Existencia de documentación de tipo técnica.

- Propuestas de intervención, anteproyectos, proyectos, trabajos de diplomas, registros estadísticos, etcétera.

- Estado del arte.

- Base de datos confiable, organizado según preceptos contemporáneos (análisis sistémico).

Es importante conocer toda la información referente al objeto de estudio, incluso la documentación que no parece necesaria en un momento puede adquirir relieve en otro, así como contar con una base de datos confiable y organizada según los preceptos contemporáneos. Se debe clasificar esta base y validarla, analizando si los períodos son ricos o pobres en información, establecer los posibles nexos y el estado existencial de la indagación.

3) Proceso de investigación histórica.

- Organizar cronológicamente el estado de la información sobre el objeto, definiendo:

- El período fundacional, fecha y contexto.

- El desarrollo cronológico del objeto:

a) Fechas o períodos significativos por eventos, fenómenos, usos o comportamientos asociados. Cronología y contexto.

b) Fechas o períodos significativos por cambios tecnológicos sustanciales (reconstrucciones, ampliaciones, demoliciones, destrucciones, etc.), cronología y contexto.

- Los períodos socioeconómicos importantes (prehispánico, colonial, republicano o actual) para la vinculación de los cambios tecnológicos y el impacto medioambiental.

- Los vacíos o lagunas presentes en la información.

-La categorización de la importancia de cada período y la planificación por prioridad en las acciones investigativas. 
- El accionar investigativo debe abarcar:

a) Revisión y búsqueda en archivos y bibliotecas.

b) Revisión y búsqueda on line.

c) Revisión, búsqueda y análisis comparativo de los resultados de las investigaciones documentales con las arqueológicas o descubrimientos fortuitos.

d) Establecimiento de nexos entre fenómenos históricos y su expresión en las fuentes.

e) Validación de las fuentes e información brindada.

f) Trabajo de campo para el conocimiento de tradiciones y leyendas asociadas.

El proceso investigativo requiere organizar cronológicamente toda la información existente para, en el análisis, detectar posibles lagunas o vacíos informativos que se deben resolver. De ahí la importancia de obtener toda la indagación sobre el objeto de estudio, sin obviar fuentes. Todo nuevo aporte histórico nos presentará, a la vez, nuevos interrogantes; y en ese ir y retornar a fuentes editadas e inéditas es como vamos despejando las incógnitas encontradas.

4) Elaboración del informe de investigación con los resultados obtenidos y finalización de la base de datos históricos sobre el objeto de estudio, quedando explícito lo siguiente:

- Fecha, época y estado del arte en el momento fundacional.

- Fechas y caracterización de los hitos constructivos y de cambio de uso.

- Desarrollo sociocultural y económico del contexto inmediato y vinculación directa o indirecta con el objeto de estudio, definiendo fechas, estado del arte y eventos asociados.

- Fechas y caracterización de procesos bioclimáticos importantes (ciclones, huracanes, terremotos y desastres naturales, en general) y su impacto en el objeto.

- Fotocopias de fuentes o referencias de estas (documentos, fotos, entrevistas, etc.).

Al elaborarse el informe investigativo, con los resultados obtenidos, concluye el proceso y se completa la base de datos históricos sobre el objeto de estudio, quedando definido, del modo más acabado posible, el acercamiento investigativo propuesto. Estaremos entonces listos para enfrentar, con éxito, cualquier intervención en el proyecto de gestión patrimonial escogido.

\section{Conclusiones}

Hasta el presente, los estudios históricos para la gestión del patrimonio edificado generalmente adolecen del análisis integral. Prima el propósito de validar soluciones tecnológicas y valoraciones histórico-culturales, útiles solamente para el momento de intervenir en un monumento. Aspectos que resultan pobres e insuficientes, al verse solo como necesarios a la hora de conservar y restaurar el patrimonio edificado, pero no se mencionan como parte fundamental en 
todo el proceso de gestión, del cual forman parte, además de su conservación, restauración, planificación, investigación, diseño, ejecución, protección, administración, uso, presentación y promoción.

El estudio del Convento de Santa Clara de Asís, sede del Cencrem, posibilitó actualizar la información sobre esta importante edificación y mostrar cómo la complejidad de la intervención y la premura en la esfera de los resultados provocaron que no se utilizaran correctamente los estudios históricos, con los criterios acordes con el fenómeno de la restauración. Además de constituir el primer caso en Cuba de un programa de gestión para la recuperación de un monumento con base en un proyecto integral de intervención, sobre el que se crean las bases de una estrategia cubana en las intervenciones para la restauración y conservación arquitectónica.

Gracias a este análisis fue posible definir una propuesta de metodología para la realización de una investigación histórica en los proyectos de gestión y manejo del patrimonio edificado. Partiendo de la definición del objeto de estudio, según la clasificación del patrimonio cultural dado por la legislación vigente, debemos analizar la situación del estado informativo que se tiene sobre aquel, para después realizar la investigación propiamente dicha siguiendo un orden cronológico. Finalizando con la elaboración del informe de los resultados obtenidos, para completar la base de datos históricos sobre el objeto tratado. Queda así definido, del modo más acabado posible, el acercamiento investigativo propuesto.

\section{Referentes bibliográficos}

Cárdenas, Eliana. La investigación histórica en los monumentos arquitectónicos, preservación y revalorización. Arquitectura y urbanismo. ISPJAE, 1981, volumen II.

Hardoy, Jorge E. y Gutman, Margarita. Impacto de la urbanización en los centros históricos iberoamericanos. Editorial Mapfre, Madrid, España, 1992.

Herrera López, Pedro A. El Convento de Santa Clara de La Habana Vieja. Cencrem, La Habana Vieja, 2006.

Jokilehto, Jukka y Fielden, Bernard. Manual para el manejo de sitios del patrimonio mundial cultural. Colcultura, Iccrom, Unesco, Icomos, Bogotá, Colombia, 1995.

La gestión, clave para la preservación y sostenibilidad del patrimonio cultural. Unesco. Representación en Perú, 2003.

Lamas, Waldo y Valdés, Oswaldo. Historia del Convento de Santa Clara de Asís, datos históricos y leyendas. Imprenta Montalvo, Cárdenas y Co., Habana, 1922.

Leal, Eusebio. Para no olvidar. Editorial Bologna, La Habana Vieja, 2001.

Memorias. Cátedra Unesco. Gestión integral del patrimonio en centros históricos. Universidad Nacional de Colombia, Sede Manizales, 2000. 
"Memorias del encuentro internacional para la formulación de entrenamiento en la gestión de las ciudades Patrimonio de la Humanidad". Iccrom, OVPM, WHC, Municipalidad de Quito, Ecuador, noviembre del 2000.

"Memorias del encuentro latinoamericano sobre museos, patrimonio y turismo cultural". Trujillo, Perú y La Paz, Bolivia, mayo 2000. Icom, Lima, Perú, 2001.

Merlín, Condesa de. Mis doce primeros años e Historia de sor Inés. Imprenta El Siglo XX, La Habana, 1922.

Pol, Francisco. Ciudad, historia, proyecto. Mopu, Madrid, 1988.

Plan de desarrollo integral. Oficina del Historiador, La Habana Vieja, 1998.

Protección del patrimonio cultural. Compilación de textos legislativos. Consejo Nacional de Patrimonio Cultural. Ministerio de Cultura, 2002.

Proyecto Cuba 81-017 PNUD-Unesco. Informe final. Biblioteca Cemcrem.

Rigol, Isabel; Rojas, Ángela y Fornés, José. "Formación en gestión de la conservación de ciudades Patrimonio de la Humanidad en Cuba”. Ponencia al Encuentro internacional para la formulación de entrenamiento en la gestión de las ciudades Patrimonio de la Humanidad. Iccrom, OVPM, WHC, Municipalidad de Quito, Ecuador, noviembre del 2000.

Rigol, Isabel. Sobre autenticidad. Memorias del simposium interamericano sobre autenticidad. US-Icomos. San Antonio, Texas, EE.UU., 1996.

Taboada Espiniella, Daniel. "Antiguo Convento de Santa Clara en La Habana Vieja. Restauración para Centro Nacional de Conservación, Restauración y Museología” (material mecanografiado). Biblioteca Cencrem.

Terán Bonilla, José A. Metodología de investigación de centros históricos. Unam, México DF, 1990.

Vera, Milet y Pontual, Virginia. "Evaluación de la gestión de las ciudades Patrimonio Mundial de América Latina. Ponencia al 5to Coloquio Internacional de la Organización de ciudades Patrimonio Mundial." Santiago de Compostela, España, 1999.

Viñueles, Graciela y Gutiérrez, Ramón. La documentación histórica en la restauración de monumentos. Cuadernos de arquitectura y conservación del patrimonio artístico. Serie Ensayos n.ํㄹ 2, México, marzo de 1976. 


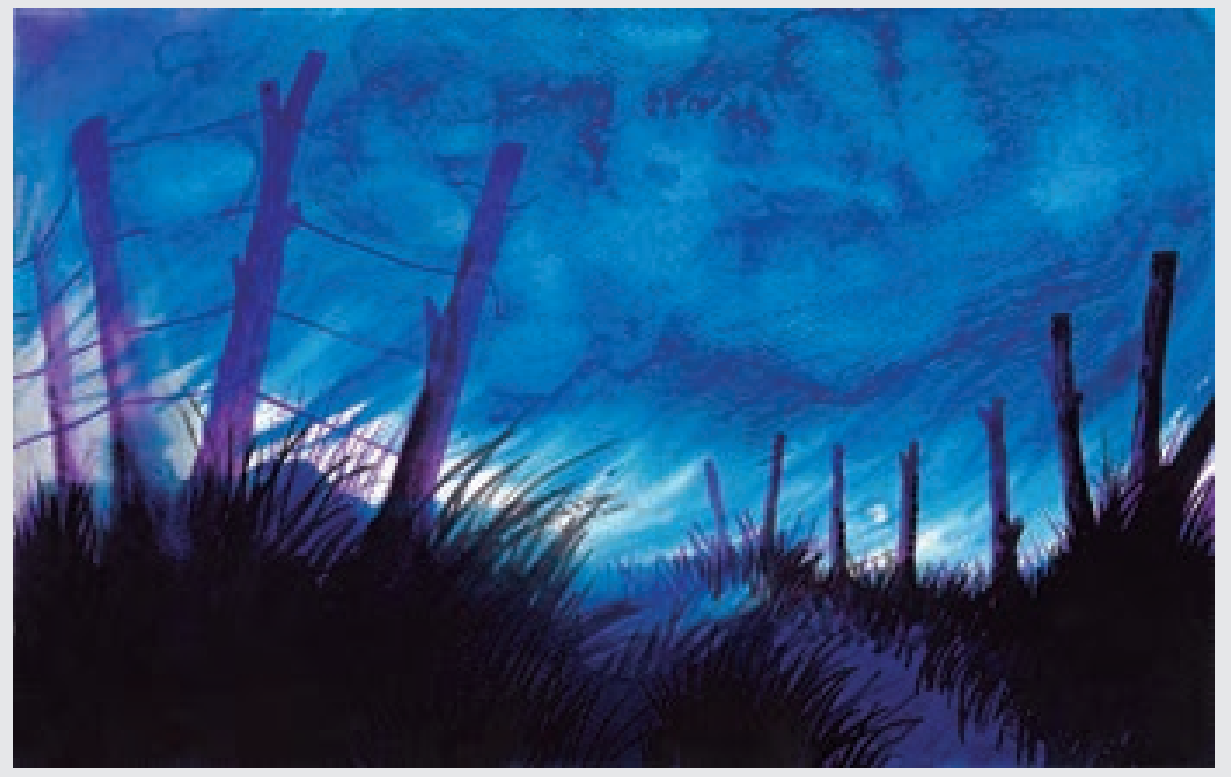

